\title{
Why do bridging veins rupture into the virtual subdural space?
}

\author{
T YAMASHIMA, RL FRIEDE \\ From the Department of Neuropathology, University of Göttingen, Göttingen, Federal Republic of Germany
}

SUMMARY Electron microscopic data on human bridging veins show thin walls of variable thickness, circumferential arrangement of collagen fibres and a lack of outer reinforcement by arachnoid trabecules, all contributory to the subdural portion of the vein being more fragile than its subarachnoid portion. These features explain the laceration of veins and the subdural location of resultant haematomas.

Most subdural haematomas due to venous bleeding have been attributed to lacerations in bridging veins. These veins form short trunks passing directly from the brain to the dura mater, almost at right angles to both. Between these two points, bridging veins take a straight course with no tortuosity to allow for the possible displacement of brain. ${ }^{1}$ Trotter $^{2}$ speculated that subdural haematomas are invariably due to trauma tearing large veins, an interpretation elaborated by Krauland. ${ }^{3}$ According to Leary, ${ }^{4}$ the common sources of subdural haematomas are ruptured bridging veins, which tend to yield at the arachnoid junction, producing small openings. Yance $^{5}$ reported two cases of subdural haematoma caused by rupture of bridging veins, and found that the torn veins were occluded by newly formed thrombotic clots. However, little attention has been given to the laceration mechanism of bridging veins.

The cranial ends of bridging veins are firmly fixed to the rigid dura mater, while the cerebral ends are attached to the movable hemisphere. The falx protects the brain from lateral displacement, but there is no protection against antero-posterior movement. Bearing this in mind, Trotter ${ }^{2}$ speculated that a sharp blow to the front or the back of the head could easily produce a substantial cerebral dislocation, causing the rupture of bridging veins. Leary ${ }^{4}$ assumed that bridging veins tear easily because their

\footnotetext{
Address for reprint requests: Tetsumori Yamashima, MD, Department of Neurosurgery, University of Kanazawa School of Medicine, Takaramachi 13-1, Kanazawa City, 920, Japan.

Address for correspondence: Reinhard L Friede, MD, Department of Neuropathology, University of Göttingen, Robert-Koch-Str. 40, D-3400 Göttingen, Federal Republic of Germany.
}

Received 26 July 1983. Accepted 14 September 1983. walls are delicate, lacking muscle fibres, with only a thin fibrous wall and a thin elastic lamina adjacent to the endothelial layer. The conclusions of these two authors, have gained wide acceptance, although there was little evidence concerning the fragility of the vein walls.

The purpose of the present communication is to provide electron microscopic data on tissue fixed in situ, which might throw some light on to the laceration mechanism of bridging veins and its relationship to the development of subdural haematoma. Special attention has been given to the differences between the subdural and subarachnoid portions of the veins. The ultrastructure of bridging veins has not been previously studied.

\section{Material and methods}

At necropsy, a steel needle was gently tapped with a hammer and pushed through the lamina cribrosa into the subarachnoid space at the medial surface of the frontal lobe. Once the subarachnoid position of its tip had been verified by aspiration of a few drops of cerebrospinal fluid, the subarachnoid space was perfused with $3.5 \%$ glutaraldehyde. After fixation in situ for $30 \mathrm{~min}$, a $6 \times 8 \mathrm{~cm}$ bone square was traced parasagittally in the frontal region. Then, a block of tissue including calvarial bone, dura mater, leptomeninges and cortex, all in their undisturbed relationship, was dissected. This block was immersed in $2.5 \%$ glutaraldehyde, and the brain tissue was completely removed from the leptomeninges, exposing bridging veins in their normal juxtaposition both to dura mater and leptomeninges. The bridging veins were subsequently dissected to demonstrate their subdural and subarachnoid portions. The following descriptions are based on the seven best-prepared specimens obtained from four patients: male aged 77 years; male aged 53 years; female aged 77 years; female aged 85 years, who had died of heart failure, liver disease, senility and abdominal tumour, respectively. 
Necropsy was performed within 5 hours of death. There were no neuropathological changes neither in the brains nor in the meninges. One specimen was postfixed in $10 \%$ formalin and embedded in paraffin. Serial sections, $10 \mu \mathrm{m}$ thick were cut parallel to the superior sagittal sinus, and stained with haematoxylin-eosin. The other six specimens were stored in cacodylate-buffered $2.5 \%$ glutaraldehyde for a few hours and postfixed in $1 \%$ osmium tetroxide buffered in $0.1 \mathrm{M}$ cacodylate for one hour. Specimens were then dehydrated and embedded in Araldite. Semithin sections were cut with a LKB ultramicrotome and stained with toluidine blue. Ultrathin sections were contrasted with uranyl-acetate and lead-citrate and were examined with a Zeiss 9S-2 electron microscope.

\section{Results}

GENERAL STRUCTURE OF THE VEIN WALLS Light microscopy Gross inspection showed that the subdural portions of the veins were embedded in the dura mater and the adjoining arachnoid membrane, while the subarachnoid portions ran parallel

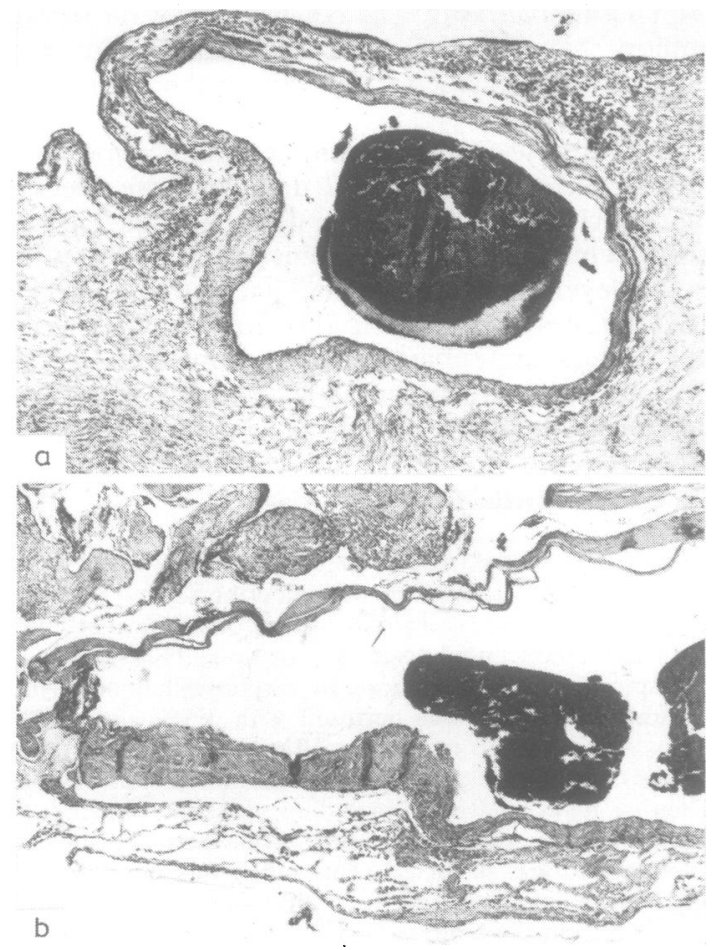

Fig 1 (a) The subarachnoid portion of the bridging vein has a wall of uniform thickness, embedded into the loose tissue of the arachnoid trabecules. (b) The walls of the subdural portion of this vein have thick, as well as extremely thin, regions, and there is no abutting arachnoid trabecular tissue reinforcing the wall from the outside.

Haematoxylin-eosin stain; $\times 18$. to the dura adhering to the arachnoid. The subdural portions of bridging veins measured $1-3 \mathrm{~mm}$ in diameter and up to $1-2 \mathrm{~cm}$ in length. Microscopically (fig 1), the thickness of the vein walls in the subdural portions varied remarkably, ranging between 10 and $600 \mu \mathrm{m}$ (fig 1b), whereas subarachnoid portions had walls of relatively uniform thickness, between 50 and $200 \mu \mathrm{m}$ (fig 1a). In both portions, the walls were composed of a layer of dense fibrous tissue encompassing the lumen and covered by thin or loose connective tissue. A single elastic lamina, up to $7 \mu \mathrm{m}$ in thickness, lay next to the endothelial lining. Many elongated cells with bilateral slender processes were scattered in the vein walls.

Fine structure Although some autolytic changes had occurred, most of the vein walls, obtained within 5 hours of death, provided useful information for electron microscopy. The lumen of bridging veins was lined with flattened endothelial cells (figs $2,4)$, covering one to several tiers of cells encompassed by basement membranes (fig $2 a$ ). The wall contained an abundance of collagen fibres and a variable number of elastic fibres, along with scattered, elongated mesenchymal cells. The external covering consisted of attenuated cells derived from either dural border cells or from arachnoid trabecules.

Texture of collagen fibres There were significant differences in the distribution of collagen fibres in the subdural (fig 4) and the subarachnoid (fig 3) portions of the vein walls. The former generally showed a loose webbing of fibres, whereas the latter had a more dense structure. The size of the electron-lucent interstitial spaces between collagen fibres indicated the potential exchange of fluids through the vessel's wall (fig 4). More than half of the collagen fibres were circumferential, the longitudinal fibres being much less abundant (fig 4).

Endothelial cells Endothelial cells (figs 2, 4) were seen in flattened or contracted states, depending upon the shape and distension of the vessel. Flattened cells were as thin as $0.2 \mu \mathrm{m}$ (fig $2 \mathrm{a}$ ), whereas contracted cells were as thick as $4.5 \mu \mathrm{m}$. The length of these cells varied, but did not usually exceed $37 \mu \mathrm{m}$. Flattened cells had a smooth surface, whereas contracted ones had irregularities and numerous cytoplasmic protrusions into the subendothelial tissue. Flattened endothelial cells had elongated nuclei with a smooth surface, while the nuclei of contracted cells showed indentations or tortuosities. Mitochondria and strands of rough endoplasmic reticulum were common, although not abundant (figs 2, 4). Mitochondria were usually spherical with an average diameter of $0.25 \mu \mathrm{m}$. Free ribosomes were dispersed throughout the cell. Sparse, small inpocketings of the plasma membrane 


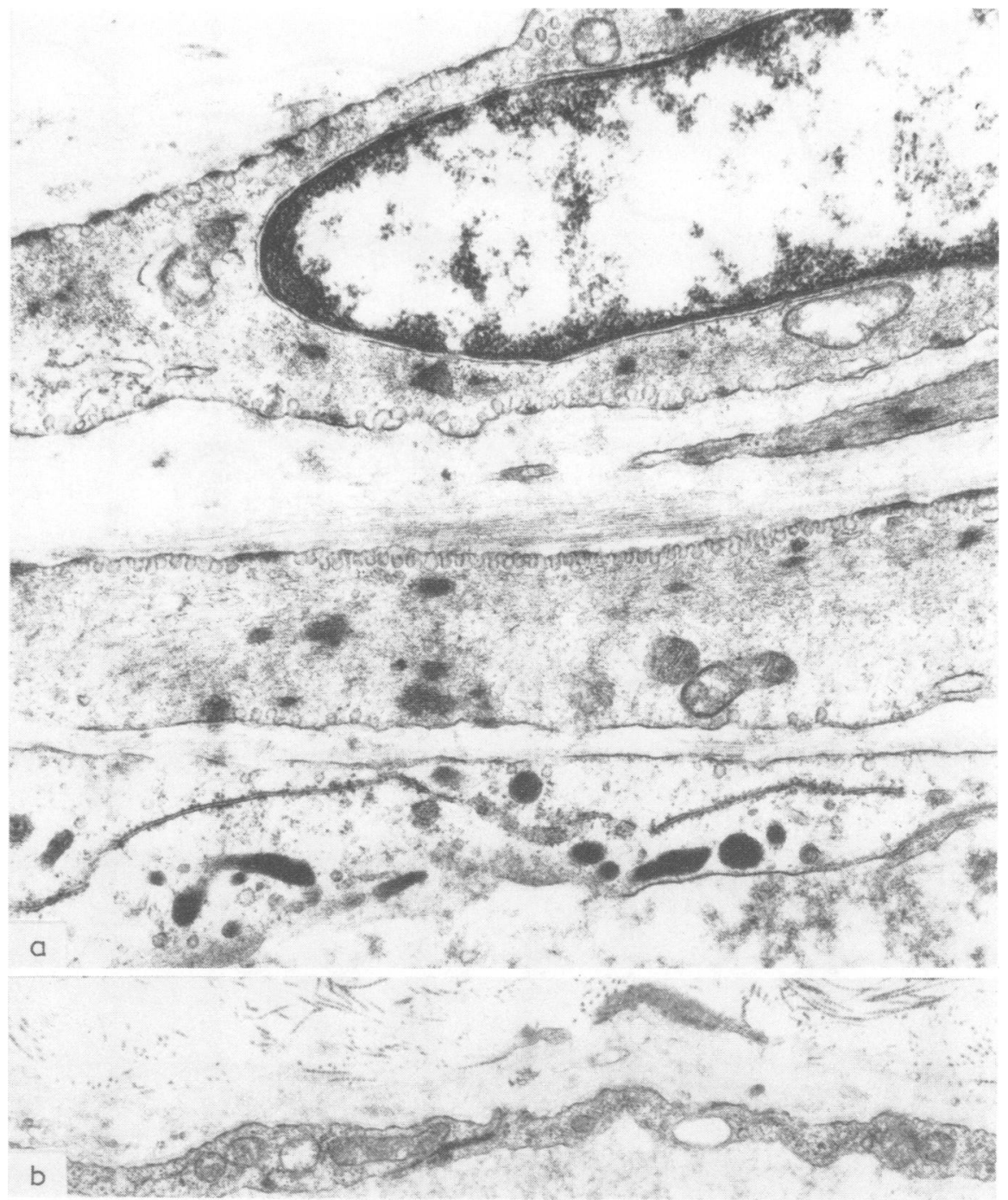

Fig 2 (a) An endothelium containing numerous Weibel-Palade bodies and three adjoining tiers of pericytic elements, identifiable as smooth muscle cells due to their dense myofilaments. Pinocytosis abounds at the abluminal surface. $\times 13$ 500. (b) An area, in which the inner lining of the vein's wall consists merely of a flattened endothelium and a thick, multilayered basement membrane containing some amorphous material. There were no adjacent layers of cells towards the fibrous wall of the vessel. $\times 13500$.

could be seen. Some of the endothelial cells contained numerous Weibel-Palade bodies, presenting as rod-shaped, oval or spherical granules with a maximum length of $0 \cdot 14$ to $1 \cdot 1 \mu \mathrm{m}$ (fig $2 \mathrm{a}$ ). Adjoining endothelial cells were linked by tight junctions amongst overlapping processes. Junctions amongst contracted cells were found between high marginal folds.
Smooth muscle cells Beneath the endothelial cells, there was a thick, multilayered basement membrane. It abutted upon one to six tiers of flat cells (fig 2a), which extended their slender processes parallel to the endothelial cells. Using the criteria set by Rhodin, ${ }^{6}$ and with no intention of pre-empting our bias, we identified these cells as smooth muscle cells rather than pericytes. The smooth muscle cells had a 


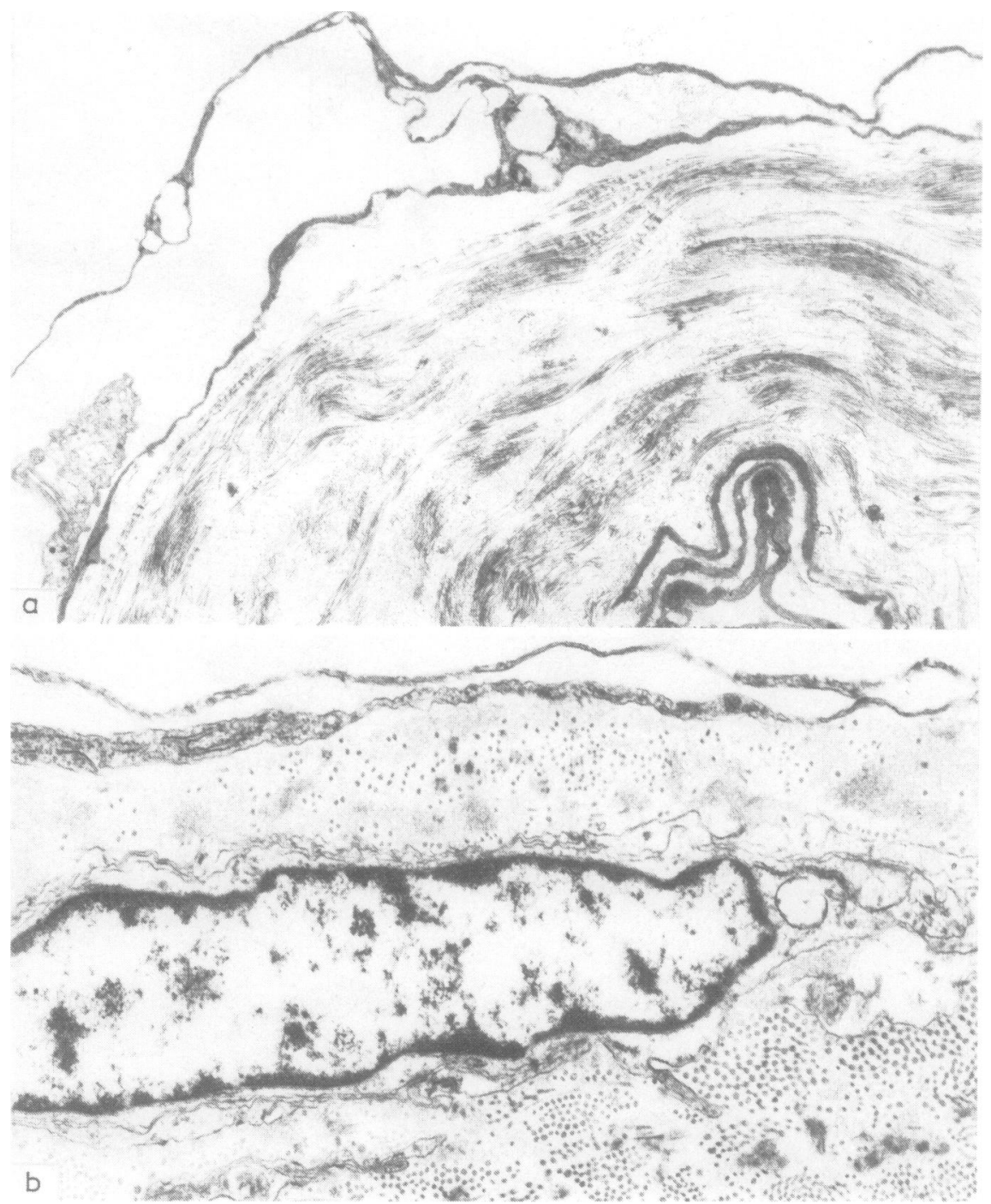

Fig 3 Features characteristic of the subarachnoid portion of bridging veins. The thick wall contains mostly circumferential collagen fibres. At its outer surface, there are scattered mesenchymal cells $(b)$ and attached arachnoid trabecular cells $(a, b)$, ofien in thicker tiers than shown here. (a) $\times 5$ 500; (b) $\times 13500$.

transverse diameter of $0 \cdot 3$ to $4 \cdot 3 \mu \mathrm{m}$ in their central portion and a length of 7 to $8 \cdot 3 \mu \mathrm{m}$. Expanded cells were flat and attenuated, whereas contracted ones showed tortuosities with protruding cytoplasm. Zones of contact between the smooth muscle cells were extremely rare (fig $2 \mathrm{a}$ ), as most cells lay separately, each having its own envelope of basement membrane. Myoendothelial junctions, in which the membrane of a smooth muscle cell contacted an endothelial cell, were quite rare. Extracellular debris was common, being embedded in the thickened zones of basement membrane (fig 2b), presumably indicating degeneration and disintegration of smooth muscle cells. Smooth muscle cells contained numerous pinocytotic vesicles, especially in their abluminal sides (fig 2a). These vesicles were even more abundant in contracted cells, also in their luminal sides. The cytoplasm contained an abundance of myofilaments and dense bodies (fig 2a), but other organelles were comparatively rare. Dense 


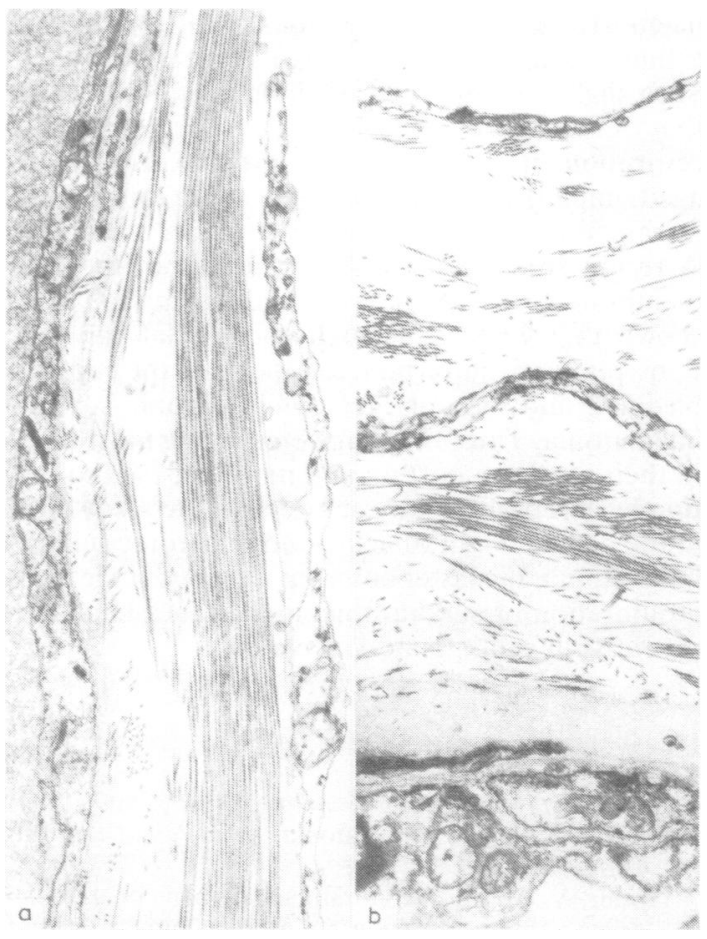

Fig 4 (a) An extremely thin portion of the wall consisting merely of a thin endothelium with basal membrane, a thin sheet of circumferential collagen fibres and a single flat cell, presumably from the dural border layer. (b) A thicker portion with a relatively thick basement membrane and a very loose collagen tissue, its fibres running mostly circumferentially, separated by two tiers of flattened cells. $\times 13500$

bodies, 0.07 to $0.50 \mu \mathrm{m}$ in diameter, were either randomly dispersed or sometimes attached to the cytoplasmic side of the plasma membrane (fig 2a). The nuclei were oval or elongated and contained granular, irregularly distributed heterochromatin, which tended to accumulate along the margin of the nuclear membrane (fig $2 a$ ). In relaxed cells, the nuclei measured $10.8 \mu \mathrm{m}$ in maximum length and $0.65 \mu \mathrm{m}$ in average width. The nuclear surface was fairly smooth with hardly any indentations. The nuclei of contracted cells showed highly wrinkled or scalloped profiles.

External surface The superficial tissue of the walls of bridging veins contained scattered mesenchymal cells with elongated nuclei and slender cytoplasmic processes. These cells were usually surrounded by a thin layer of amorphous substance including a few collagen and elastic fibres (fig 3 ). The subdural portion of the bridging veins was covered by a discontinuous layer of attenuated cell bodies with few organelles (figs $4 a, b)$ and/or a few dural border cells permitting free communication between the potential subdural space and the vein wall. The external coat of the subarachnoid portion consisted of a relatively constant layer of attenuated cell bodies (figs $3 a, b)$, lacking tight intercellular junctions. This external coat was tightly reinforced by arachnoid trabecular cells. These were elongated with slender cytoplasmic processes and abundant swollen mitochondria. Their elongated nuclei had marginal accumulations of heterochromatin and a few nucleoli (fig 3 ). The processes of arachnoid trabecular cells lay upon arachnoid trabecules composed of collagen fibrils and elastic fibres found within large amounts of fuzzy material (fig 3a).

\section{Discussion}

Our light microscopic data are mostly consistent with the description given by Leary. ${ }^{4}$ The thickness of the bridging vein walls varied remarkably in the subdural portion, the thinnest part measuring as little as $10 \mu \mathrm{m}$. In the subarachnoid portion, however, the walls had a fairly constant thickness of 50 to $200 \mu \mathrm{m}$. Our electron microscopic examination further showed that the walls of the veins had a compact distribution of collagen in the subarachnoid portion, whereas in the subdural portion, the collagen fibres were only loosely woven. Moreover, the subdural portion of bridging veins lacked the tight reinforcement of arachnoid trabecular cells, and there were only scattered attenuated dural border cells. The arrangement of collagen fibres varied from one portion to the other, but there was always a tendency for circumferential fibres to prevail compared with longitudinal ones, particularly in the subdural part. This pattern would render the vein's wall more resistant to distension while reducing its resistance to traction. Bridging veins, furthermore, had their ends firmly embedded into the rigid dura mater, while their cerebral extensions, reinforced by arachnoid trabecules, were attached to the comparatively movable hemisphere. All these features imply that bridging veins are more fragile in the subdural portion than in the subarachnoid portion. Indeed, the differences in the structure of the two portions of the bridging vein were so striking that one is less inclined to ask: "Why do bridging veins rupture easily?", but rather: "Why isn't the rupture of bridging veins more common, especially in the subdural portions?".

The antero-posterior acceleration or deceleration of the head can easily cause traction of the bridging veins, and they will rupture at their weakest point, that is, in the subdural space. Cerebral atrophy will accentuate this mechanism. 
Rhodin ${ }^{6}$ observed smooth muscle cells with intimate intercellular linkage in small collecting veins. The smooth muscle cells (or pericytes, respectively) of bridging veins were not interconnected. They may, therefore, tighten the vascular wall rather than constrict the vascular lumen. Degeneration or disintegration of the smooth muscle cells due to ageing, implied by cellular debris, may conceivably weaken the walls.

Schachenmayr and Friede ${ }^{7}$ showed that the socalled subdural space in man is the result of a cleavage artefact produced by the tearing of dural border cells within the dura arachnoid interface layer. The absence of collagen from this interface facilitates the cleavage between dura and arachnoid, and it could also weaken the reinforcement of bridging veins traversing the interface layer.

Conceivably, a physiological increase of intravenous pressure, for example from defaecation or coughing, could also cause leakage at the weakest portions of the bridging veins, even in the absence of trauma. One of the authors of the present paper (RLF) has observed, over many years and in many patients, that thin, fresh subdural haemorrhages found at necropsy often correlate with terminal external cardiac massage causing violent compression of the thorax or even rib fractures. In conclusion, one may state that not only sudden acceleration or deceleration of the head but also sudden increases in venous pressure can lead to an augmentation of tension, especially at the subdural portion of bridging veins, thus inducing subdural bleeding.

\section{PATHOGENESIS OF CHRONIC SUBDURAL \\ HAEMATOMA}

The initial bleeding in subdural haematoma is commonly attributed to the rupture of bridging veins, although other sources have also been claimed including dense capillary networks of the internal dural layer, ${ }^{8}$ parasagittal venular vessels extending from the calvaria to the dura, ${ }^{9}$ pial veins ${ }^{10}$ or, recently, subarachnoid arteries. ${ }^{11}$ Several authors have thought chronic subdural haematoma to be the consequence of a single event of bleeding caused by mechanical trauma. Trotter ${ }^{2}$ was the first to regard the rupture of bridging veins as the cause of chronic subdural haematoma. Christensen ${ }^{12}$ observed, during surgery, firm clots of blood around bridging veins. The veins bled profusely after the clots had been removed. Krauland ${ }^{3}$ observed stumps of torn bridging veins in chronic subdural haematoma. Patients with chronic subdural haematoma often have a history of trivial trauma.

Thin subdural neomembranes are a common finding during routine necropsy in the absence of other lesions. ${ }^{13}$ Conceivably, slight subdural haemorrhage or transudation, with or without trauma, is more common than generally assumed. Such slight subdural extravastations of blood within the dural border layers may undergo complete resorption, if organisation is not impaired. In the aged, impaired organisation may cause formation of neomembranes, which have a tendency to rebleed. A recent study ${ }^{14}$ on the fine structure of the outer membranes of chronic subdural haematomas has shown that leakage through endothelial gap junctions plays an important role both in the repeated bleeding and the enlargement of chronic subdural haematoma. Thus, bridging veins, being most fragile in their subdural portion and more exposed to traction by cerebral atrophy, may constitute a key factor in the development of acute and of chronic subdural haematoma, the latter entering a vicious circle of the proliferation of subdural neomembranes leading to repetitive bleeding, and vice versa.

\section{References}

1 Baker AB. Subdural hematoma. Arch Pathol Lab Med 1938;26:535-59.

${ }^{2}$ Trotter W. Chronic subdural haemorrhage of traumatic origin, and its relation to pachymeningitis hemorrhagica interna. Br J Surg 1914;2:271-91.

${ }^{3}$ Krauland W. Uber die Quellen des akuten und chronischen subduralen Hämatoms. Stuttgart: Thieme, 1961.

${ }^{4}$ Leary T. Subdural or intradural hemorrhages. Arch Pathol Lab Med 1939;28:808-20.

${ }^{5}$ Yance BM. Ruptures of surface blood vessels on cerebral hemispheres as a cause of subdural hemorrhage. Arch Surg 1950;61:992-1006.

${ }^{6}$ Rhodin JAG. Ultrastructure of mammalian venous capillaries, venules, and small collecting veins. $J$ Ultrastruct Res 1968;25:452-500.

'Schachenmayr W, Friede RL. The origin of subdural neomembranes. I. Fine structure of the duraarachnoid interface in man. Am $J$ Pathol 1978;92:53-68.

${ }^{8}$ Hannah JA. The aetiology of subdural hematoma. An anatomical and pathological study. J Nerve Ment Dis 1936;84:169-86.

${ }^{9}$ Krempien B. Über die Blutungsquelle der Durahämatome. Ein Beitrag zur Pachymeningeosis haemorrhagica interna. Virchows Arch (Pathol Anat) 1968;343:210-29.

${ }^{10}$ Rand CW. Chronic subdural hematoma. Report of seven cases. Arch Surg 1927;14:1136-65.

"Shenkin HA. Acute subdural hematoma. J Neurosurg 1982;57:254-7.

${ }^{12}$ Christensen E. Studies on chronic subdural hematoma. Acta Psychiatr Scand 1944;19:69-148.

${ }^{13}$ Friede RL. Incidence and distribution of neomembranes of dura mater. J Neurol Neurosurg Psychiatry 1971;34:439-46. 
${ }^{14}$ Yamashima, T, Yamamoto S, Friede RL. The role of endothelial gap junctions in the enlargement of chronic subdural hematomas. J Neurosurg 1983;59: (in press). 